\title{
La función de asesoría para la mejora de la enseñanza y el aprendizaje en la práctica profesional de un orientador de zona. Un estudio de caso
}

\section{Advising as a tool to improve teaching and learning in an Educational Counselor's professional practice. A case study}

\author{
Jesús Domingo Segovia* \\ jdomingo@ugr.es \\ Juan de Dios Fernández Gálvez* \\ juandefg@gmail.com \\ Beatriz Barrero Fernández** \\ beabarrero@ugr.es \\ * Universidad de Granada, España \\ * Universidad de Jaén, España
}

\section{Resumen:}

El reto de la mejora exige buenas prácticas docentes y unos determinados modelos de asesoría. En Andalucía, están institucionalizados unos equipos de zona de apoyo para Educación Infantil y Primaria: los Equipos de Orientación Educativa. Están compuestos en su mayoría por orientadores escolares. Se encargan tanto de la orientación educativa como de la promoción y apoyo al desarrollo de diferentes programas educativos. Pueden actuar como colegas críticos, asesores y agentes clave para la mejora de los procesos de enseñanza y aprendizaje. El artículo presenta los resultados de un estudio de caso, seleccionado por ser un claro ejemplo de buenas prácticas de asesoría para la mejora de los resultados desde la orientación. El estudio se ha desarrollado a lo largo de cuatro años. Utilizando un enfoque bio-

\begin{abstract}
:
Any desire to improve involves good teaching practices and certain models of counseling. In Andalusia, a few teams of support for pre-school and primary education are institutionalized: Educational Guidance Teams. They consist mainly of school counselors. They are in charge both of educational counseling and the promotion and support of different educational programs. They can act as advisors, critical colleagues, and key elements in the improvement of teaching and learning processes. The article presents the results of a case study which was selected because it clearly links the improvement of results form orientation with good practice in counseling. The study has been developed over four years. It uses a narrative biographical approach through in-depth interviews for the purpose of making its
\end{abstract}


La función de asesoría para la mejora de la enseñanza y el aprendizaje en la práctica profesional de un orientador de zona. Un estudio de caso

Jesús Domingo Segovia, Juan de Dios Fernández Gálvez y Beatriz Barrero Fernández

gráfico narrativo, mediante entrevistas en profundidad para hacer emerger su teoría fundamentada. Ofrece las principales claves de actuación como asesor para la mejora desde la orientación: incidir sobre el currículum y con un modelo de trabajo colaborativo, constructivista, comunitario y de procesos.

Palabras clave:

Orientación Educativa, Asesoramiento, Mejora, Socio-constructivismo. theoretical foundations more grounded. It explains some key ideas, coming from the field of orientation, which advisors could introduce in their professional practice: giving the curriculum a more prominent role and developing that curriculum through a model of work which is collaborative, constructivist, community, and based on processes.

\section{Key words:}

Educational Guidance, Counseling, Improvement, Socio-constructionist.

\section{Résumé :}

Afin de relever ce défi de perfectionnement, il est nécessaire d'élaborer de bonnes méthodes d'enseignement et un modèle type de conseiller. En Andalousie quelques équipes de soutien scolaire sont institutionnalisées en maternelle et en primaire: ce sont les équipes d'orientation scolaire. Elles sont principalement composées de conseillers d'orientation. Elles sont chargées de promouvoir et de soutenir le développement de différents programmes éducatifs et de lıorientation pédagogique. Ses membres peuvent être à la fois conseillers, collègues critiques et intervenants : rôles clés pour l'amélioration des méthodes d`apprentissage et d`enseignement. L`article présente les conclusions d`une étude de cas, sélectionnée pour être l'exemple même des conseils à suivre par le conseiller afin de relever le défi posé : c`est-à-dire "l lamélioration des résultats par lıorientation ». L`étude s'est déroulée sur quatre ans. Elle se base sur approche biographique/narrative et se sert d'entretiens poussés afin d'en faire émerger une théorie. L'étude propose les principales attitudes à suivre par le conseiller dans le but de participer à l'amélioration de l'enseignement par l'orientation: peser sur le programme scolaire, adopter une attitude de collaboration, constructiviste, communautaire et technique.

\section{Mots clés:}

Orientation pédagogique, conseil, amélioration, Socioconstructivisme.

Fecha de recepción: 1-6-2013

Fecha de aceptación: 21-10-2013

\section{Presentación y fundamentación del problema de investigación}

La orientación educativa en España, tras un largo recorrido de aciertos y trabajos en los márgenes del sistema ha logrado consolidar un lugar, un prestigio y un espacio profesional. De acuerdo con Bisquerra (2000, 2008) se ha seguido paralelamente una evolución conceptual y ampliación del espacio profesional. Una vez que la escolarización se ha universalizado, fue desplegándose su ámbito de influencia hacia el campo del aprendizaje, dando lugar a lo que hoy se conoce como Orientación Escolar o Educativa (Bisquerra, 2000; Santana, 2003, Nieto y Botías, 2000). 
A lo largo del proceso, con sus luces y sus sombras, se ha ido evolucionando de lo clínico y casi en los márgenes a ser considerado un factor de calidad en educación (Bolívar, 2008), a modo de apoyo necesario para alcanzar los diez indicadores que inciden en la construcción de una educación de calidad para todos en el siglo XXI (Braslavsky, 2006).

Para llevar a cabo un adecuado desarrollo de la misma se crearon una serie de servicios, destinados a realizar intervenciones de modo puntual. Con el tiempo estos sistemas de apoyo han ido evolucionando hasta alcanzar un desarrollo más adecuado a la situación educativa, creándose los Equipos de Orientación Educativa (EOE) como se conocen en Andalucía, o Equipos de Orientación Educativa y Psicopedagógica (EOEP) en otros puntos del Estado, y desde enfoques de modelos de programas van implicándose en objetivos más concretos y en situaciones más generales, desarrollando funciones cercanas al constructivismo, a la colaboración, la contextualización, a la visión estratégica de procesos, etc. (Escudero y Moreno, 1992; Guarro, 2005) y al actuar con módulo en una creciente estructura colaborativa en red (Nieto y Portela, 2001). Desde esta perspectiva, los servicios pueden centrarse en dar apoyo para la mejora de la calidad educativa (Bolívar, 2008, p. 238), ayudando al profesorado, en la labor docente, y al alumnado, en problemas vinculados con el aprendizaje.

Hoy día, todos los centros de Educación Infantil y Primaria disponen de la ayuda de un orientador, aunque sea de manera intermitentemente; por ejemplo: dos mañanas a la semana y una tarde al mes, dependiendo de la densidad de población a la que tenga que atender el servicio de apoyo externo (EOE). Pese a la discontinuidad de actuación, estos profesionales son considerados un dispositivo valorado y necesario.

Ahora bien, las funciones asignadas a estos profesionales son motivo de constante controversia e incesante debate. En torno a ellas continúan las discrepancias pues se desarrollan en un complejo entramado de situaciones, tareas, programas, acciones y escenarios, todos ellos importantes y con frecuencias urgentes, que bloquean en no pocas ocasiones las posibilidades de flexibilidad o las opciones de priorización que darían racionalidad, integridad y cohesión a su tarea.

A pesar de que la normativa (andaluza en este caso: Decreto 213/1995) establece una serie de funciones relativas al trabajo del orientador, estas son tan generales que dan lugar a heterogéneas interpretaciones por 
La función de asesoría para la mejora de la enseñanza y el aprendizaje en la práctica profesional de un orientador de zona. Un estudio de caso

Jesús Domingo Segovia, Juan de Dios Fernández Gálvez y Beatriz Barrero Fernández

parte de los profesionales del campo y a no pocos desacuerdos interpretativos de las mismas.

Algunas de ellas a pesar de ser vitales para la mejora, suelen quedar no cubiertas, en contraposición de otras mucho más demandas y menos efectivas. Una de las funciones más demandadas, e incluso valoradas, a estos agentes y menos cubierta es la función de asesoría (Boza, 2001). Los orientadores como asesores, conforman instrumentos trascendentes a través de sus funciones como agentes de cambio, de innovación, de consultor y formador de formadores (Boza, Toscano y Salas, 2007). Llegándose a afirmar que los orientadores como asesores, podrían e incluso deberían ocupar un papel de gran importancia en los centros, como agentes ligados a los procesos de mejora (Bisquerra, 2008; Hopkins, 2007, Guarro, 2005, Lago y Onrubia, 2011).

La asesoría aterriza en los centros escolares -provista de autoridadpara promover procesos de mejora en contextos educativos, dirigida a solucionar problemas a alumnos/as con dificultades de aprendizaje, para poco a poco ir ganando terreno y llegar a ser concebida como un factor clave en los procesos de mejora del sistema educativo (Hopkins, 2007; Guarro, 2005).

Es labor del asesor guiar y orientar el conocimiento y las estrategias para que los docentes puedan elaborar sus propios proyectos de mejora, siendo ellos mismos los que los desarrollen y los evalúen. Asesorar consiste en "ayudar a mejorar las formas de enseñar y aprender" (Monereo y Pozo, 2005), de forma que implica conocer los procesos de enseñanza y aprendizaje en el foco de la mejora educativa. La principal meta del asesoramiento es que cada centro, profesor y comunidad desarrollen procesos orientados y con sentido en aras de alcanzar la mejora educativa (Hopkins, 2007).

El rol del asesor se va conformando a medida que estos profesionales se van involucrando en experiencias de asesoramiento, existiendo toda una serie de factores (percepción, fusión contextualización...) implicados en dicho proceso que modularían su construcción de manera dinámica y contextual (Guarro, 2005, p.300).

El verdadero sentido del asesoramiento "educativo" o "psicopedagógico", no es otro que ayudar a escuelas y profesorado a que hagan efectiva la garantía del buen aprendizaje para todos los estudiantes (Domingo y Hernández, 2008). El asesoramiento es una práctica controvertida, compleja y dinámica en la que no está todo tan atado (Domingo, 2005, 
p. 248). Este no constituye un único tipo de prácticas de apoyo, sino más bien un conjunto heterogéneo de ellas que se desarrollan en diferentes escenarios con propósitos, agentes y estrategias diversas (Guarro, 2005, p.295), entre los que los profesionales de la orientación pueden perfectamente llevarlo a la práctica.

Se debe olvidar de la idea de entender al asesor psicopedagógico como aquel que, con cierta marginalidad, contribuye a "tapar agujeros" (Bolívar, 2008, p.238). De este modo, la función de asesoría y el desempeño del rol asesor, lejos de interpretaciones tradicionales de consejo técnico o mera información, conlleva otros matices que le dotan de nuevos sentidos. Fullan (2002, p.207) la precisa bien cuando afirma que esta labor consiste en desarrollar la capacidad, la motivación, y el compromiso necesarios para implicarse en los procesos de mejora.

La asesoría debe generar procesos de mejora que conviertan al centro escolar en un lugar donde el aprendizaje no sólo es una meta, sino una práctica capaz de asegurar unos niveles educativos deseables para todos los alumnos (Bolívar, 2007, p.10). Lo que lleva implícito fomentar en los centros y equipos docentes procesos reflexivos sobre la práctica real y sus consecuencias de cara al buen aprendizaje de todos, lo que debería ser motor para hacer recapacitar, repensar viejas prácticas y certezas y atreverse a andar otros caminos de innovación y sentido común profesional.

En definitiva, ayudar a los docentes a introducir mejoras de su práctica diaria en las aulas, apoyando a estos a que varíen la metodología que emplean por prácticas más eficaces, integradoras y educativas (Lago y Onrubia, 2008). En definitiva, no se trata de que el asesor aporte soluciones elaboradas a problemas particulares -como si fuera un especialista- sino que debe adoptar el papel de facilitador, de apoyo, en procesos para que la escuela identifique y explore lo que hace.

Esbozada la teoría, también es pertinente tomar conciencia de la dificultad de la propuesta en las circunstancias actuales del ejercicio de la orientación educativa (Boza, Toscano y Salas, 2007). No se trataría, pues, de añadir funciones y tareas a una profesión ya saturada de exigencias y normativa, sino promover una reflexión crítica y una redimensión de la misma. Sabemos que con las prácticas tradiciones y comunes no se impacta en el núcleo duro de la mejora (De la Oliva, Martín y Vélaz de Medrano, 2005), luego algo habrá que hacer. Y, en este punto de debate, los profesionales de la orientación, pueden contribuir y promover la in- 
novación en la práctica diaria (Martínez, Krischesky y García, 2010; Domingo, 2006; Hargreaves y Fink, 2006; Sink, 2009; Janson, Stone y Clark, 2009) a poco que se sientan agentes educativos comprometidos con la mejora del centro, asistan al profesorado en sus procesos de cambio metodológico, colaboren con los docentes para mejorar el desarrollo integral del alumnado, y trabajen en estrecho vínculo con el equipo directivo para desarrollar un proyecto conjunto de comunidad profesional de aprendizaje.

\section{La investigación: Qué opina un orientador sobre sus labores de asesoramiento}

\subsection{Objetivo}

Mostrada la importancia que supone que los profesionales de la orientación desarrollen la función asesora, y conocida dificultad de que se practique significativamente (Boza, Toscano y Salas, 2007), la investigación pretende mostrar sintéticamente las claves profesionales de un caso que está haciendo posible una redimensión de las funciones de los Equipos de Orientación Educativa, con la intención de indagar y obtener claves para ubicar y comprender la realidad y posibilidades de los orientadores de equipo como asesores, desde un enfoque procesual y socioconstructivista, y dinamizadores en su zona y centros de influencia de cambios "educativos" con sentido y utilidad social.

Obviamente, la contribución que se haga a partir del estudio y análisis del caso no es extrapolable ni pretende mostrar "el modelo ideal" de actuación. Sino obtener un conjunto de evidencias, matices y pistas de cómo actúa un profesional y en qué cree, que pueden ser de gran ayuda para comprender una opción de desempeño profesional, con unas determinadas posibilidades y necesidades de formación, que bien pueden tomarse en consideración a la hora de comprender, formar y promover maneras de desempeño de determinadas y muy importantes funciones de apoyo.

Su opción, puede ser una buena experiencia a tener presente para aquellos que ven tremendamente dificultoso actuar como agente de apoyo a la mejora. Y, tomada con suficiente distancia y tiento, puede ser bastante ilustradora para promover reflexión profesional entre los que no 
crean que deben ejercerse por los orientadores estas funciones y los que han tenido la suerte de vivenciarlas y experimentar sus frutos.

\subsection{Presentación del caso objeto de estudio}

Con tal fin, se necesitaba un orientador de EOE (Equipo de Orientación Educativa) que desarrollase cotidiana y prioritariamente estas labores de asesoramiento. Y, como se toma partido por un determinado modelo de asesoría, coincidente con lo señalado en el punto anterior, se necesitaba alguien que practicase dicho modelo.

Se seleccionó un orientador con una dilatada trayectoria profesional, con amplio reconocimiento -entre los colegas de profesión y por las autoridades educativas- como ejemplo de buenas prácticas en labores de asesoría y apoyo a procesos de innovación. Algunos datos relevantes son: miembro histórico de los MRPs, ponente asiduo en actividades formativas en CEP para profesorado y orientadores, coordinador provincial de estos servicios de apoyo en dos mandatos, con procesos de ida y vuelta a su zona de actuación, y que realizó su tesis doctoral precisamente sobre la función asesora de los EOEs (Fernández, 2005) y con una visión crítica de los mismos (Fernández, 2006).

Entre la profesión se le considera como un referente por su conocimiento psicopedagógico, su experiencia profesional en el ámbito de la orientación y su cercanía para con el profesorado y los padres. Ciertamente, como cualquier estudio de caso, es único, pues ha vivido y experimentado una larga trayectoria profesional en unos determinados momentos y contextos, lo que ha hecho evolucionar a lo largo de diferentes modelos de actuación, hasta calar en enfoques que priorizan las funciones de acompañamiento y asesoría psicopedagógica.

El caso es más interesante si cabe, si se sabe que el orientador ha Ilegado a estos principios y a esta manera de actuar en plena madurez profesional, después de recorrer múltiples vicisitudes en un proceso de desarrollo (más de treinta años), y que afirma fundamentar su práctica como fruto de un proceso de ir contrastando personalmente sus prácticas con los resultados obtenidos y con los principios pedagógicos y sociales que considera más potentes, a modo de proceso de investigación-acción.

Se le reconoce una "buena" práctica profesional, caracterizada por dejar a un lado la expertez del diagnóstico y la intervención clínica y puntual de modo reactivo, por la prevención y las actuaciones holísticas 
con carácter proactivo. Su preocupación está en cómo hacer posible en cada caso y contexto el pasar de los "problemas" a las "problemáticas y ámbitos de mejora", que más que "intervenir en" se interesa por "trabajar con", desarrollando al máximo las potencialidades de los profesionales con los que trabaja, en torno a un proyecto o programa conjunto de acción. Y que enmarca su práctica profesional dentro de un enfoque eminentemente educativo (Sanz Oro, 1996), con énfasis en un currículum democrático y una escuela inclusiva, en la que minimiza su rol de terapeuta, maximizado el de educador, trabajando de apoyo a los equipos para favorecer procesos reflexivos y colaborativos a nivel de profesorado, familias y alumnado.

La presencia de estas características y principios en su práctica profesional, al coincidir con los lineamientos que se pretenden estudiar, hacen del caso un buen objeto de estudio.

\subsection{Método}

La finalidad de la investigación no es describir una panorámica ni pretende extrapolar datos y propuestas, sino comprender determinadas dinámicas. Por ello se opta por una perspectiva cualitativa de investigación (Flick, 2004; Albert Gómez, 2006). Y dentro de ella, se adopta el estudio de caso único (Stake, 2010).

Consientes del sentido poder de la teoría fundamentada (en la práctica profesional), se llega a ella desde una perspectiva biográfico-narrativa (Bolívar, Domingo y Fernández, 2001). Es decir, desde la descripción de una serie de relatos de experiencia profesional, se accede al conocimiento práctico elaborado por un orientador desde su reflexión y el debate generado para mostrar su práctica real y el sentido de la misma, justificando su opción por el desempeño de nuevos roles y maneras de hacer enfatizando su función asesora.

La investigación biográfico-narrativa ayuda a comprender e interpretar la realidad educativa y constituye una potente herramienta, especialmente pertinente, para entrar en el mundo de la identidad, de los significados y del saber práctico, de las claves cotidianas presentes en los procesos de interrelación, identificación y reconstrucción personal y cultural (Acebes, 2001). Y con este diálogo reflexivo, de acuerdo con la teoría fundamentada (Strauss \& Corbin, 2008; Birks \& Mills, 2011; Charmaz, 2006), se accede, reconstruye y produce conocimiento que 
ayuda a comprender e interpretar la realidad educativa. Investigar desde este marco metodológico, permite profundizar sobre ciertas cuestiones primordiales, mediante la estimulación de la reflexión, como un medio válido para construir conocimiento en la investigación educativa (Bolívar, Domingo y Fernández, 2001, p.56).

Se utiliza, como mejor instrumento para la recogida de evidencias, una cascada de profundización comprensiva, mediante un ciclo de 24 entrevistas en profundidad desarrolladas a lo largo de un proceso de cuatro años. En esta secuencia, las evidencias y relatos, a medida que iban siendo analizados, les eran devueltos al informante clave para que los revisase, profundizase y validase, entrando a debate o profundización de determinados elementos, con nuevas reflexiones y relatos... Y así hasta llegar a saturar la información.

En los ciclos de entrevistas y las reflexiones profesionales emergentes del debate en torno a ellas se ha seguido un proceso de profundización sucesiva y de triangulación secuencial. Para el análisis de la información, se utilizó, primeramente un sistema de categorías emergentes de la teoría (apegado a las funciones asignadas a estos equipos, los modelos de trabajo, las temáticas más usuales a los campos de acción de un orientador...). Con ello se garantizaba tratar todos los aspectos claves que el conocimiento pedagógico señala como relevantes y sobre los que se debía tratar en los relatos y entrevistas. Seguidamente, fueron los propios relatos los que marcaron los contenidos y centros de interés, que se configuraron en las "unidades de significado" definitivas (frases o párrafos que abordaban con pleno sentido una idea dentro del objeto de estudio). Para ello se utilizó, tanto la parrilla de categorías inicial como el análisis de frecuencia de palabras y campos semánticos, así como de los árboles de contenido mediante el programa Nvivo.

La veracidad de la información se consiguió mediante un proceso de negociación y clarificación, las cascadas de profundización y el volver reiteradamente a los puntos en blanco o que necesitaban de mayor debate. El informante disponía en todo momento de las transcripciones y primeros análisis y el resultado final fue consensuado dialógicamente. Finalmente, se trianguló esta perspectiva biográfico-narrativa, una entrevista con la Inspección Educativa de zona y mediante cuatro grupos de discusión (uno por cada centro en el que desempeña su labor este orientador), conformados por entre 3 y 4 profesores del centro y los miembros de su equipo directivo. 
La función de asesoría para la mejora de la enseñanza y el aprendizaje en la práctica profesional de un orientador de zona. Un estudio de caso

Jesús Domingo Segovia, Juan de Dios Fernández Gálvez y Beatriz Barrero Fernández

\section{Resultados}

Los resultados informan cómo el informante clave describe, entiende y desarrolla su trabajo. Estos datos -extraídos de la suma de todos los relatos a lo largo del proceso de investigación- muestran cómo debe ser el trabajo del orientador en los centros de Educación Infantil y Primaria desde su conocimiento profesional. La reconstrucción dialógica de estas historias ilustra cuáles son las dificultades, los puntos de tensión, las zonas de riesgo, etc. del proceso de asesoramiento que ha vivido y desarrollado. Seguidamente se presentan cuáles son, a su juicio, las claves de trabajo de los orientadores en la tarea asesora; así como las ideas, los prejuicios e inquietudes que tienen los docentes en relación a la organización y gestión de estos servicios.

a) Frecuencia de palabras y campos semánticos más representados. Estar para ser en el cambio metodológico

En primer lugar las palabras más usadas (con pleno sentido) han sido "centro", y "todos", seguidas de "temas", "niños" y "estar". Si cabe, el campo semántico del centro es predominante, puesto que a este término habría que añadir también la frecuencia de "Colegios", con 296 apariciones más en las narraciones. Lo que ratifica su visión comunitaria y nos puede indicar que para el sujeto de estudio la globalidad va por encima del trabajo individualizado. Procurando que todos sean conocedores y participes de un mismo proceso de desarrollo.

"...todos los centros están llevando agenda". (ent12)

"...entre todos los profesionales aprobamos ese documento". (ent13)

"...es responsabilidad de todos los tutores". (ent13)

"...estamos todos de acuerdo en desarrollar esta idea en los centros, porque todos hemos estado hablando lo mismo". (ent10) 
La función de asesoría para la mejora de la enseñanza y el aprendizaje en la práctica profesional de un orientador de zona. Un estudio de caso Jesús Domingo Segovia, Juan de Dios Fernández Gálvez y Beatriz Barrero Fernández

Tabla 1

\begin{tabular}{lccl}
\hline \multicolumn{3}{l}{ 10 palabras con mayor } & frecuencia \\
\hline Palabra & Conteo & Porcentaje ponderado (\%) & Palabras similares \\
\hline centro & 1709 & 0,93 & centro, centros \\
todos & 1216 & 0,66 & todo, todos \\
temas & 812 & 0,44 & tema, temas \\
niños & 806 & 0,44 & niño, niños \\
estás & 719 & 0,39 & está, estás \\
hacer & 620 & 0,34 & hacer \\
sistemático & 612 & 0,33 & sistema, sistematizar \\
equipo & 579 & 0,31 & equipo, equipos \\
trabajo & 570 & 0,31 & trabajo, trabajos \\
colegios & 478 & 0,26 & colegio, colegios \\
profesor & 474 & 0,26 & profesor, profesores \\
\hline
\end{tabular}

Las palabras "niños", "temas" suele usarlas en frases en las que se deja entrever su idea de trabajar y de entender la labor orientadora como parte importante del proceso educativo, no como apéndices o anexos. Señala en este sentido que su acción tiene más sentido si ayuda a hacer posible la integración de programas y acciones en propuestas de trabajo globales, inespecíficas y colaborativas, enfatizando todo en el trabajando en temáticas que de verdad incidan directamente en el mejor aprendizaje de los niños. Sin este nexo con la realidad del aula y con impacto en los resultados (en sentido amplio) de aprendizaje de todos, su actuación está infrautilizada y mal orientada.

Otra idea principal, repetida a lo largo de casi todas las entrevistas es la importancia de que el orientador de referencia esté en los centros. "Estar" no solo físicamente en el despacho asignado del centro para trabajar. Sino "estar" para "ser" interno, parte implicada, miembro de un equipo. Es un estar proactivo y participante sobre un tema de interés y con potencial de mejora. Lo que implica estar en reuniones, en los pasillos, en los recreos, en los aparcamientos.... Estar en aquel lugar donde el profesorado hable, se sienta relajado para exteriorizar cuáles son sus verdaderas sensibilidades, sus miedos, sus retos. En este sentido, afirma que

"Pudiera parecer una pérdida de tiempo estar en las entradas y salidas, o en otros espacios informales, pero es en ellos en donde se cimenta "su 
La función de asesoría para la mejora de la enseñanza y el aprendizaje en la práctica profesional de un orientador de zona. Un estudio de caso

Jesús Domingo Segovia, Juan de Dios Fernández Gálvez y Beatriz Barrero Fernández

cercanía" y da opciones hablar cotidianamente. Una vez ganado el estar, es cuando se puede empezar a ser y a influir (ent18).

Los orientadores deben entrar y salir a la par con el profesorado, para generar sentido de pertenencia, de cotidianeidad, de cercanía, de apego al mismo centro...

"Que te vean que sales y entras cuando ellos. La permanencia en el centro debe ser de 9 a 2. Olvidemos los horarios flexibles para este profesional. La gente no puede dudar si a las once y media aun será pronto para encontrar al orientador. Este el día que le toque tiene que estar a las 9 y salir a las 2, y si por alguna causa debe abandonar el centro debe comunicarlo con antelación al profesorado" (ent07).

"Cuando perciban, el colectivo del profesorado, que tu presencia allí es buena y necesaria y ocurra algún problema se van a acordar de él, lo van a solicitar para que les ayudes. Ese es el momento clave para que el orientador verdaderamente actúe como el experto educativo que es" (ent09).

Acompañando a esta idea, es también importante para este orientador llevar a cabo un trabajo sistemático "en" los centros, y que se desarrolle a lo largo de la jornada escolar. No es sólo estar e interaccionar; hay que ser y hacer posible, para lo que es fundamental la planificación estratégica y tener muy claro lo que es fundamental e imprescindible, de lo que se puede negociar, aplazar o cambiar.

Los orientadores de zona educativa deben asumir el papel de externos pero no acatar el significado de este concepto. "Yo soy externo, pero mi implicación va a ser tanta que tienes que pensar que soy parte de tu centro" (ent03). Esa es, a su juicio, la verdadera filosofía que un orientador debe abanderar. Este profesional debe implicarse en los asuntos del centro como lo que son, como asuntos "propios" en los que se siente comprometido. Con sentido de pertenencia, de identificación y comunitario, como resalta reiteradamente, reclamando que se construya y dinamice un verdadero compromiso entre la tarea orientadora y el desarrollo de los centros.

La implicación que se demanda a este profesional opina que debe ser sentida realmente como propia. De este modo, los éxitos y los fracasos, las dudas y las propuestas son del colectivo; aunque, sin duda, su actuación ha influido en que esto sea o no posible. 
Reclama también que debe actuar, tanto generando como participando, en debates educativos que pongan en duda muchos de los procedimientos arcaicos que siguen vigentes en los centros y que vayan experimentándose pequeños cambios metodológicos, dentro de las zonas de desarrollo próximo del colectivo y del propio centro educativo.

"En el horizonte está el cambio metodológico. Cambiando pequeñas piezas de la metodología y de la evaluación es posible ir aproximándose sin demasiado ruido a un necesario cambio curricular para ir a un currículum más potente, enraizado y competencial para todos" (ent20).

Está plenamente convencido de que hay muchos procedimientos que hay que desaprender, implícitos que hay que explicitar, "por supuestos" que hay que cuestionar... Y, para ello, defiende el debate pedagógico y la argumentación crítica, sobre el currículum, sobre lo que acontece en el día a día y las razones esgrimidas para darle sentido, en un contexto de trabajo en equipo, como caldo de cultivo de la mejora, la profesionalización y el cambio cultural.

\section{b) Frecuencias y relación de categorías. Colaboración, rumbo y estrategia}

La categoría con mayor número de referencias lo largo de todas las entrevistas ha sido "principios, claves y declaraciones" con una total de 210 unidades de significado codificadas a lo largo de las narraciones. Por lo que se puede considerar la categoría "estrella" del análisis. Y alerta de que verdaderamente se ha puesto en marcha la teoría fundamentada de este orientador para presentar y argumentar sus relatos. En ella se pueden conocer orientaciones sobre qué actitud deben tener los orientadores; cómo ha de desarrollarse su trabajo; qué otros agentes intervienen en este proceso u otras circunstancias del entorno que favorecen o dificultan su tarea.

Las frecuencias de categorías y sus relaciones, muestran como el trabajo colaborativo es una idea-fuerza que se repite a lo largo de las entrevistas y que se va entretejiendo con el resto de los argumentos y situaciones. Argumento que queda claramente explicitado en el siguiente argumento: "Podemos decir que la colaboración es el ingrediente principal de esta relación. Cuando en los centros existen verdaderas redes colaborativas todo empieza a ser posible" (ent12). De este modo resalta 
La función de asesoría para la mejora de la enseñanza y el aprendizaje en la práctica profesional de un orientador de zona. Un estudio de caso

Jesús Domingo Segovia, Juan de Dios Fernández Gálvez y Beatriz Barrero Fernández

la idea de que cualquier objetivo es más fácil de alcanzar cuando el esfuerzo de muchos está orientado hacia ese mismo fin. Las relaciones colaborativas provocan que los procesos de cambio sean revisados y consensuados constantemente, lo que produce un desarrollo mucho más profundo.

El trabajo del orientador muchas veces gira en torno a generar o plantear propuestas de trabajo. Propuestas que no sean demasiado innovadoras pero que sí provoquen ruptura. Desde la teoría sabemos que cuando una propuesta es implantada, trasladada desde otro contexto o, promovida sin tener de referencia cómo es el terreno, su probabilidad de agarre es muy pequeña. Contar con el apoyo de una gran parte del profesorado hará que ellos se sientan partícipes, y lo sean, de procesos de cambio.

El sujeto estudiado tiene en mente unas líneas de trabajo que cree que son sensibles a cualquier centro, pero estas no tiene la perspectiva fija de donde van a terminar. Es decir, se sabe cómo empiezan pero no se saben hasta donde las hará posibles el colectivo docente. En definitiva, todo orientador debe tener en mente unas líneas de trabajo, para no llegar de cero a los centros, sino marcar unos límites sobre los que sería interesante trabajar. Ayudar a alcanzar y visualizar los resultados que se puedan obtener de estos procesos pueden ser claves para ir ganando sentido y generando un discurso comunitario de que merece la pena experimentar esa "pequeña" revolución.

Enlazada con esta idea, advierte que es también muy importante que el orientador tenga un norte (de buena escuela, buenas metodologías y buenos aprendizajes) y ser fiel (aunque con flexibilidad) a unos principios de actuación procesuales, democráticos, socio-constructivistas, dialógicos, participativos y con apoyaturas técnicas potentes... Las problemáticas de los centros pueden turbar los procesos de mejora y generar múltiples altibajos que pueden Ilegar a desanimar, confundir o sacar del camino planteado. Este profesional debe estar ahí también a pesar de ser complicado:

"Cuántas veces no se sabe realmente hacia dónde se camina y en ello el orientador debe tener tanto una forma de trabajar bien consensuada como un norte alcanzable (aquí y ahora, con este equipo). No es dirigir y erigirse como salvador, sino saber manejar la situación y apoyar iniciativas interesantes, así como actuar como abogado del diablo que ayuda a que prevalezca 
el sentido común profesional. El orientador debe mantener su idea firme y no olvidar cuál es la meta, aunque siempre deba estar abierto a argumentar, escuchar, integrar..." (ent18).

Desde esta perspectiva "pedagógica" o "educativa", no tiene mucho sentido que dedique la totalidad de su tiempo a realizar diagnósticos, informes, programas y actuaciones que no calen en el núcleo duro de la mejora... Estas tareas, sin duda son también parte de su trabajo, pero hay que tener cuidado con ellas para no quedar enredadas en sus rutinas y dinámicas que lo alejan de lo cotidiano.

A lo largo de la mayoría de los encuentros el sujeto de estudio piensa que para mejorar los ambientes escolares, es necesario disponer de una amalgama de recursos tanto humanos como materiales que permitan acompañar el desarrollo de propuestas de trabajo. Y, para ello, propone trabajar directamente con el profesorado, sin olvidar los "líderes" pedagógicos del centro y que van a ser los verdaderos agentes que faciliten el despliegue de nuevas iniciativas. Estos otros agentes pueden ser los coordinadores de ciclo, la jefatura de estudio, la dirección e incluso las familias.

\section{c) Integración de resultados: Principios de actuación}

A modo de síntesis de las reflexiones y argumentos en torno a los relatos de experiencia, se recolectan las claves que, a juicio del orientador, pueden ser utilizadas por el orientador en su trabajo diario dentro de los centros a los que acompaña y apoya. Los resultados informan que las claves que se enumeran seguidamente potencian, ayudan y favorecen la tarea asesora promotora de la idea de mejora que se defiende en el trabajo. Estas trazan lineamientos que pueden ser oportunos para disponer, orquestar y tener en cuenta, pero con la precaución de que ni son ni universales, ni uniformes, ni a prueba de centros y profesorado:

- Poseer una metodología y filosofía de trabajo y tenerlas bien interiorizadas, para concretarlas en un determinado modelo de actuación adaptado a cada contexto.

- Se considera esencial el contacto directo con jefatura de estudios y/o dirección. Delegando o demandando la autoridad que corresponde a este puesto en la escuela.

- La labor orientadora ha de alejarse del aspecto técnico de intervención puntual, apostando cotidiana y estratégicamente por la perspectiva constructivista, la base del asesoramiento educativo, 
y de procesos con un enfoque de resolución de problemas que promueva que la repuesta sea tomada por el colectivo docente.

- No olvidar su tarea principal, ser un mediador de conocimiento, facilitando la formación y el acompañamiento necesario al colectivo docente para que sea éste quien elabore, desarrolle y evalúe propuestas, planes, programas y tareas.

- La colaboración: el orientador está "obligado" a promover el trabajo cooperativo y a colaborar con el centro en todas las actuaciones de carácter educativo, aportando sus conocimientos, su implicación y su ayuda en todo lo que el centro considere oportuno.

- El rol que desempeña este profesional debe girar en torno a la participación, la integración, la dinamización de procesos, etc. desde estos parámetros de actuación se pueden lograr varias cosas: de una parte, se favorece a la derrota de prejuicios asociados a estos servicios de ayuda y, de otra, se redimensionan las funciones tradicionales que suelen asociarse a este colectivo - diagnosticador e interventor- por otras más pertinentes de mediador y asesor pedagógico.

- Son imprescindibles también la interrelación y la flexibilidad.

d) La otra mirada: Voces de triangulación

Las palabras más usadas a lo largo de las entrevistas grupales informan que, aún con pequeños matices, obvios por otra parte, los conceptos, temáticas y discursos son bastante consistentes con los obtenidos del orientador. Predominan palabras como centro, niños, todos, está, trabajo, equipo... y son muy frecuentes las palabras en colectivo. Destaca también como aparece en $18^{\circ}$ lugar, la palabra "diferente". Lo que puede ser todo un signo del sentir y actuar de este modelo de orientación con énfasis en asesoría "comunitaria".

La puesta en práctica de estos principios del modo como lo viene haciendo este "particular orientador", como afirma el Inspector de Zona, puede ser una de la claves principales de la existencia de cambios de diferente índole: en la creación de acuerdos e implicación de los agentes educativos alumnado, profesorado y familias en un proceso educativo con envergadura hacia la innovación y mejora, en la creación de un proyecto conjunto e incluso en los resultados escolares. 
La función de asesoría para la mejora de la enseñanza y el aprendizaje en la práctica profesional de un orientador de zona. Un estudio de caso Jesús Domingo Segovia, Juan de Dios Fernández Gálvez y Beatriz Barrero Fernández

En Pueblo hay un orientador que tiene una concepción de la orientación muy colaborativa. Entiende la orientación desde la colaboración, desde el trabajo, de cooperación y colaboración con los centros [...] Y ha sido capaz de aglutinar y de trabajar de manera conjunta, de crear un equipo de trabajo entorno a núcleos temáticos en los centros. Que va más allá de lo que es la propia orientación, incidiendo en el propio Proyecto Educativo de los centros (entIE)

Los profesionales de la zona de actuación, también reiteran la importancia de esta labor del orientador en asesoría y como dinamizador de procesos de mejora en coordinación con los equipos directivos. Éstos avalan la propuesta de trabajo y forma de actuar, señalando de manera rotunda que:

"Es único. Su implicación y cercanía, hablando de lo que más nos importa y haciéndonos ver qué hacemos y qué resultados tiene en el aprendizaje, nos lleva a que también nos enganchemos en el carro" (gdC3).

"Es una persona implicada en su trabajo y lo trasmite y sabe tirar del carro, enganchar a los otros profesores, y llevarlos a donde quiere" (gdC4).

"Prefiero este modelo de actuación al de otros orientadores que hemos tenido. Porque es participativo y se implica en el centro. Aunque tenga el defecto de que no entra tanto en el diagnóstico y la clasificación de los niños, que él no lo ve" (gdC2).

"Una persona con inquietud que crea también inquietud, y eso nos hace crecer, $y$ eso es positivo. Aunque tengamos que en determinados momentos decirle ' $X$, necesitamos frenar. La gente necesita tiempo de ir asimilando los temas'. Pero yo pienso que es necesario también que nos hagan pensar y sistematizar cosas, evaluar resultados, echarnos para adelante..." (gdC1)

\section{Discusión y conclusión}

El primer lugar es necesario matizar que las afirmaciones obtenidas, pese a las limitaciones y certezas que la rodean, deben ser entendidas como resultados "no extrapolables ni generalizables". Este tipo de in- 
La función de asesoría para la mejora de la enseñanza y el aprendizaje en la práctica profesional de un orientador de zona. Un estudio de caso

Jesús Domingo Segovia, Juan de Dios Fernández Gálvez y Beatriz Barrero Fernández

vestigaciones se realizan sin intención de establecer leyes universales y generalizables, pues como establece Stake (2010) cuando se emprenden este tipo de estudios lo único que interesa es conocer bien una realidad particular. Eso sí, el rigor acometido en la construcción discursiva a lo largo del proceso de recolección, análisis y discusión de las evidencias del caso es posible vislumbrar atisbos de esperanza en la reconstrucción de la labor asesora de uno de los servicios de apoyo a la escuela con mayor prospectiva y posibilidades.

La mejora escolar es un reto necesario que debe ser abordado por todos los centros, si bien deben estar y sentirse acompañados en el proceso. Existen multitud de caminos por los que llegar a la innovación y múltiples también las opciones para acompañar en cada momento esta travesía. Lo que es harto complejo, dadas la complejidad del proceso, de las variables contextuales y de la situación ciertamente no bien resuelta sobre la articulación de los servicios de apoyo (supervisión, formación y asesoría/acompañamiento), en un modelo de autonomía de centros capaz de garantizar para todos un buen aprendizaje.

Ahora bien, en este punto de reflexión, no está nada mal ofrecer "buenas prácticas" que, llegado el momento se atrevan a ir más allá del espacio "ganado y reconocido" en que ha venido desarrollándose durante años la orientación: trabaja cotidiana y sistemáticamente en los márgenes o sin entrar en el núcleo duro, aunque de vez en cuando se permita ir sembrando resquicios (cuando le dejaban) de prácticas orientadas y potenciadoras de la mejora escolar; por otras apuestas más holísticas y apegadas a la realidad de las aulas.

Parece, a partir de la teoría fundamentada de este orientador, que se empiezan a tener evidencias que dan respuesta afirmativa a nuestro principal interrogante, ¿el orientador puede ser asesor?. Las evidencias del estudio, en concordancia con otros trabajos (Boza, Toscano y Salas, 2007), afirman que un orientador de zona, no sólo puede desempeñar labores de asesoría, sino que - de acuerdo con las historias de vida de sujeto estudiado (experimentado y bien valorado por parte del profesorado)- debería ser la función más importante de su trabajo.

Según afirma el informante, coincidiendo con toda esta línea de reflexión actual, los orientadores -desde modelos colaborativos, comunicativos, procesuales y socio-constructivistas de actuación- contribuyen significativamente a que la escuela sea una buena escuela. Su labor puede incidir directa o indirectamente, favoreciendo la creación de entornos de 
aprendizaje ricos pedagógicamente hablando. Apoyados en la idea de "acompañar" el desarrollo de comunidades profesionales de aprendizaje (Escudero, 2011), su acción -como mediador cognitivo- puede ayudar a desdramatizar los procesos de cambio (Santana, 2010). Al tiempo que apoyar que se vaya generando una cultura de cambio y de corresponsabilidad, al incidir recurrentemente en procesos dialécticos y en aspectos nucleares y sistémicos que doten a la cultura del centro de mayor cohesión con actuaciones colaborativas, alejadas de la individualidad (Hargreaves y Fink, 2006; Bolívar 2012; Fullan, 2002; Ainscow, 2005). El orientador, apoyando procesos de debate pedagógico y de búsqueda de coherencia curricular y cambio metodológico, puede ofrecer herramientas a -y desde- la jefatura de estudios para que se constituyan y llenen de contenido equipos y grupos diversos que trabajen conjuntamente para alcanzar el desarrollo profesional de los docentes y, por ende, la mejora educativa.

Es momento, pues, para sacar a debate la idea de que los orientadores -desarrollando labores de asesoramiento- pueden ser elementos significativos de mejora y profesionalización docente que ayuden al profesorado y los equipos directivos a orquestar procesos, proyectos comunes y compromisos institucionales desde tesituras colaborativas, apoyadas en proyectos preocupados y sensibles por la mejora educativa para todos y entre todos (Escudero, 2006). En este sentido, Sink (2009) afirma que una de las tareas más importantes que debe realizar el orientador en los centros es "acompañar al profesorado en los procesos de mejora y recopilar y divulgar buenas experiencias". Se trata de potenciar un empoderamiento de la labor del orientador como promotor de la mejora educativa y poner en valor su capacidad y su actuación para llegar a conseguir el cambio educativo deseado. Estos agentes, en algunos casos externos, se presentan como una pieza clave en la mejora del proceso educativo (Aguaded, Rus y Ávila, 2010, p. 450).

La tesis que defiende este trabajo, el orientador puede y debe ser asesor, tomada con la oportuna prudencia, supone un salto cualitativo que contrasta con la práctica habitual, ya consolidada y con un sólido prestigio, mucho más técnica y en los márgenes, que ha sido ampliamente descrita y cuestionada -en cuanto a su potencial de incidir en el núcleo duro del cambio- en otros trabajos (Boza, 2001; Del Rincón, 2001; Fernández Gálvez, 2006; Guarro y Hernández, 2002; Badía, Mauri y Monereo, 2004; Hernández, Santana y Cruz, 2007; Botías, 2008). Mientras que coincide con los planteamientos de redimensión de la profesión, 
La función de asesoría para la mejora de la enseñanza y el aprendizaje en la práctica profesional de un orientador de zona. Un estudio de caso

Jesús Domingo Segovia, Juan de Dios Fernández Gálvez y Beatriz Barrero Fernández

con muchas más presencia de la función asesora, desde las orientaciones más actuales y potentes (ASCA, 2008; Domingo, 2006; Lago y Onrubia, 2008; Martín y Onrubia, 2011; Monereo y Pozo, 2005).

En definitiva, este estudio de caso, puede haber captado uno de esos instantes, y poner en valor los aportes de un ejemplo vivo de cómo puede posicionarse un profesional de la orientación, tras haber desarrollado toda una trayectoria de implicación, debate y reflexión sobre su práctica. Sin duda, su aporte bien merece detenerse un instante, ponerlo sobre la mesa y repensar qué son y cómo podrían redimensionarse determinadas funciones de los orientadores para impactar realmente en el desarrollo curricular, profesional e institucional.

\section{Referencias bibliográficas}

A.S.C.A. (2008). School Counselor Competencies. American School Counselor Association (ASCA). Available from: http://www.schoolcounselor.org/files/SCCompetencies.pdf

Aceves, J. (2001). Experiencia biográfica y acción colectiva en identidades emergentes. Espiral, 7 (20), 11-38.

Aguaded, C. Rus, A y Ávila, J.A. (2010). Equipos de Orientación Educativa en la Comunidad andaluza: análisis de la situación actual en la provincia de Huelva. Electronic Journal of Research in Educational Psychology, 8, 429-456.

Ainscow, M. (2005). El próximo gran reto: la mejora de la escuela inclusiva. Ponencia presentada en el International Congress on School Effectiveness and improvement, enero. Barcelona.

Albert Gómez, M. J. (2006). La investigación educativa. Madrid: McGraw Hill.

Badía, A., Mauri. T. y Monereo, C. (coords.) (2004). La práctica psicopedagógica en educación formal. Barcelona: UOC.

Birks, M., \& Mills, J. (2011). Grounded theory: A practical guide. London: SAGE

Bisquerra, R. (2008). Orientación psicopedagógica: áreas y funciones. En VV.AA. Funciones del Departamento de Orientación. (p. 11-39). Madrid: Ministerio de Educación Política Social y Deporte.

Bisquerra, R. (Coord.) (2000). Modelos de orientación e intervención psicopedagógica. Barcelona: Praxis.

Bolívar, A. (2007). Los centros como Comunidades Profesionales de aprendizaje. (Modulo 1: El centro como contexto de innovación), en Programa de Formación en Asesoría Pedagógica (Curso de Formación especializada en centros educativos). Madrid: MEC/CNICE. Disponible en: http://www.ite.educacion.es/formacion/materiales/89/ $\mathrm{cd} / \mathrm{m} 1 / 1 . \mathrm{htm}$

Bolívar, A. (2008). Orientación y Educación para la ciudadanía. En R. Bisquerra (Coord.) Funciones del Departamento de Orientación. Ministerio de Educación, Política Social y Deporte: Instituto Superior de Formación y Recursos en Red para el Profesorado. 235-266. 
La función de asesoría para la mejora de la enseñanza y el aprendizaje en la práctica profesional de un orientador de zona. Un estudio de caso Jesús Domingo Segovia, Juan de Dios Fernández Gálvez y Beatriz Barrero Fernández

Bolívar, A. (2012). Assesoria para la melhoria da escola e do trabalho na sala de aula. En A. Bolívar. Melhrar os processsos e os resultados educativos. O que nos ensina a investigação. (p.159-190) Oporto: Editor Fundação Manuel Leão.

Bolívar, A; Domingo, J., y Fernández, M. (2001). La investigación biográfico-narrativa en educación. Enfoque y metodología. Madrid: La Muralla, S.A.

Botías, F. (2008). Estudio de la función externa de apoyo educativo: El caso de los Equipos de Orientación Educativa y Psicopedagógica en la Región de Murcia. Murcia: Universidad de Murcia (tesis doctoral inédita).

Boza, A. (2001). Los equipos de orientación de sector. Funciones y modelos de intervención. Revista Española de Orientación y Psicopedagogía, 12 (1), 50-71.

Boza, A., Toscano, M. y Salas, M. (2007). ¿Qué es lo que hace un orientador?. Roles y funciones del orientador en Educación Secundaria. XXI. Revista de Educación, 9, 111-131.

Braslavsky, C. (2006). Diez factores para una educación de calidad para todos en el siglo XXI. REICE: Revista Electrónica Iberoamericana sobre Calidad, Eficacia y Cambio en Educación, 4 (2), 84-101.

Charmaz, K. (2006). Constructing grounded theory: A practical guide through qualitative analysis. Los Angeles, CA: Sage.

De la Oliva, D.; Martín, E. y Vélaz de Medrano, C. (2005). Caracterización y valoración de los modelos de intervención psicopedagógica en centros de educación secundaria. En C. Monereo y J.I. Pozo (coords.). La práctica del asesoramiento educativo a examen. Barcelona: Graó.

Del Rincón, B. (ed.) (2001). Presente y futuro del trabajo psicopedagógico. Barcelona: Ariel.

Domingo, J. (2005). Aportaciones de la investigación biográfico-narrativa al conocimiento de la práctica asesora. En C. Monereo, y I. Pozo, (Coords.). La práctica del asesoramiento educativo a examen. Barcelona: Graó.

Domingo, J. (2006). Los Departamentos de Orientación en la mejora cualitativa de la Educación Secundaria. Revista de Educación, 339, 97-118, http://www.revistaeducacion.mec.es/re339/re339a07.pdf

Domingo, J. y Hernández, V. (2008). Una mirada crítica a la función asesora desde los servicios de apoyo externo. Recorrido y prospectiva. Olhar de profesor, 10 (2), 6380. Disponible en: http://www.uepg.br/olhardeprofessor/pdf/revista111_artigo04.pdf

Escudero, J. M. (2006). Compartir propósitos y responsabilidades para una mejora democrática de la educación. Revista de Educación, 339, 19-41.

Escudero, J.M. (2011). Los centros escolares como espacios de aprendizaje y de desarrollo profesional de los docentes. En M.T. González (coord.). Innovaciones en el gobierno y la gestión de los centros escolares. (p. 117-142) Madrid: Síntesis.

Escudero, J.M. y Moreno, J.M. (1992). El asesoramiento a centros educativos. Estudio evaluativo de los Equipos Psicopedagógicos de la Comunidad de Madrid. Madrid, CAM.

Fernández, J.D. (2005). Buscando la magia del mago sin magia. Granada: Grupo Editorial Universitario.

Fernández, J.D. (2006). Memoria sobre las funciones propias de los equipos de orientación educativa. Moción casi a la totalidad de la práctica. En Actas del I Congreso Internacional de Orientación Educativa de Andalucía, Granada. 
La función de asesoría para la mejora de la enseñanza y el aprendizaje en la práctica profesional de un orientador de zona. Un estudio de caso

Jesús Domingo Segovia, Juan de Dios Fernández Gálvez y Beatriz Barrero Fernández

Flick, U. (2004). Introducción a la investigación cualitativa. Madrid: Morata.

Fullan, M. (2002). Los nuevos significados del cambio en la educación. Barcelona: Octaedro.

Guarro, A. (2005). Los procesos de cambio educativo en una sociedad compleja. Diseño, desarrollo e innovación del currículum. Madrid: Pirámide.

Guarro, A. y Hernández, V.M. (2002). El apoyo externo a los centros educativos: necesario e incomprendido. Educar, 30, 121-138.

Hargreaves, A. y Fink, D. (2006). Estrategias de cambio y mejora en educación caracterizadas por su relevancia, difusión y continuidad en el tiempo. Revista de Educación, 339, 43-58. Disponible en: http://dialnet.unirioja.es/servlet/articulo?codigo=2057197

Hernández, V.M., Santana, L. y Cruz, A. (2007). El asesoramiento de los Equipos de Orientación Educativa y Psicopedagógica (EOEP) en el Contexto de los centros educativos. Un estudio cualitativo. Revista de Investigación Educativa, 25 (2), 287-304

Hopkins, D. (2007). Every school a great school. Realizing the potencial of system leadership. New York: McGraw Hill/Open University Press.

Janson, C.; Stone, C. \& Clark, M. A. (2009). Stretching Leadership: a Distributed Perspective for School Counselor Leaders. Professional School Counseling, 13 (2), 98-106.

Lago, J.R. y Onrubia, J. (2008). Una estrategia general de asesoramiento para la mejora de la práctica educativa Profesorado. Revista de Currículum y formación del profesorado, 12 (1), 1-13. Disponible en http://www.ugr.es/ recfpro/rev121COL5.pdf

Lago, J.R. y Onrubia, J. (2011). Asesoramiento psicopedagógico y mejora de la práctica educativa. Barcelona: Horsori.

Martín, E. y Onrubia, J. (coords.) (2011). Orientación educativa. Procesos de innovación y mejora de la enseñanza. Barcelona: Graò.

Martínez-Garrido, C., Krichesky, G., y García Barrera, A. (2010). El orientador como agente de cambio. Revista Iberoamericana de Educación, 54, 107-122

Monereo, C. y Pozo, J.I. (coords.) (2005). La práctica del asesoramiento educativo a examen. Barcelona: Graó.

Nieto, J.M. y Botías, F. (2000). Los equipos de orientación educativa y psicopedagógica: el asesoramiento a centros escolares desde un análisis institucional. Barcelona: Ariel.

Nieto, J.M. y Portela, A. (2001). La cooperación entre agentes de innovación educativa: Formas y elementos básicos. Profesorado, Revista de currículum y formación del profesorado. 5 (1), 1-16. Disponible en: http://www.ugr.es/ recfpro/rev121ART5.pdf

Santana, L.E. (2003), Orientación educativa e intervención psicopedagógica. Cambian los tiempos, cambian las responsabilidades profesionales. Madrid: Pirámide

Santana, L.E. (2010). La innovación educativa: un desafío para los orientadores como agentes promotores de las iniciativas de cambio. Revista Española de Orientación y Psicopedagogía, 21, (2), 261-270.

Sanz Oro, R. (1996). Evaluación de programas en orientación educativa. Madrid: Pirámide. Sink, C. (2009). School Counselors as Accountability Leaders: Another Call for Action. Professional School Counseling. Available from: http://www.thefreelibrary.com/

Stake, R.E. (2010). Qualitative Research: Studying How Things Work. New York: The Guilford Press.

Strauss, A., \& Corbin, J. (2008). Basics of qualitative research: Grounded theory procedures and techniques (3rd ed.). Thousand Oaks, CA: Sage. 